\title{
When Imagination Becomes a Disease: Dealing With Hypochondriacal Patients in Clinical Practice
}

\author{
David Mobley, MD, ${ }^{1}$ Neil H. Baum, MD, ${ }^{2}$ Ashley Beattie, MD, ${ }^{3}$ Charles Nemeroff, MD, $\mathrm{PhD}^{4}$ \\ ${ }^{1}$ Department of Urology, Baylor College of Medicine, Houston, TX ${ }^{2}$ Department of Urology, Tulane University School of Medicine, New \\ Orleans, LA ${ }^{3}$ Department of Psychiatry, Medical University of South Carolina College of Medicine, Charleston, SC ${ }^{4}$ Department of \\ Psychiatry, University of Texas School of Medicine, Austin, TX
}

Hypochondriacal patients are always a challenge. They require an overabundance of physicians' time; they are responsible for excessive healthcare dollars; they demand extensive and often unnecessary testing; they make repeated visits for negligible issues; they are in search of a new medication when previous medications did not help or had adverse side effects; and they are rarely satisfied when no definitive diagnosis or treatment is provided. They frequently require excessive telephone time with the physician and ancillary staff.

Many physicians fail to convince hypochondriacal patients they have nothing to worry about. These patients often feel they are sensitive to medications. They frequently claim to have a high pain tolerance so that if they have any symptoms, the symptoms must represent something serious. When hypochondriacal patients visit a new physician for the first time, they often comment that the new physician is their last resort and that they are placing their full faith and trust in the new doctor. These patients often complain that all their previous doctors have failed them.

Hypochondriacal patients also tend to overuse the emergency department, frustrating emergency personnel who can find no source of their symptoms. Imaging and laboratory tests ordered in the emergency department are negative. More medications are frequently prescribed in the emergency department.

Despite all efforts, hypochondriacal patients can rarely be convinced that what physicians are telling them is accurate. Because they remain convinced they have an as-yet-to-bediagnosed malady, psychological or psychiatric intervention is a consideration that hypochondriacal patients rarely accept.

\section{DEFINITIONS AND HISTORY OF HYPOCHONDRIASIS}

First known to be used in English in 1671, the Greek term hypochondria refers to the soft tissue area under the ribs. In ancient times, melancholy was thought to arise from the liver, gallbladder, or spleen or from the soft tissues behind the ribs, hence the term hypochondria. ${ }^{1}$

The definition in the Merriam-Webster Dictionary is "extreme depression of mind or spirits often centered on imaginary physical ailments." 2 According to the Mayo Clinic, "illness anxiety disorder, sometimes called hypochondriasis or health anxiety, is worrying excessively that you are or may become seriously ill." 3 The Cambridge
Dictionary defines hypochondria as "unnecessary anxiety about and attention to health." ${ }^{\mathrm{Dr}}$ Gene Stollerman, Chair of Medicine at The University of Tennessee, had a benign description of hypochondriacal patients; he referred to these individuals as "the worried well" (personal communication).

\section{SOMATIC SYMPTOM DISORDER AND ILLNESS ANXIETY DISORDER}

The term hypochondriasis was eliminated from the Diagnostic and Statistical Manual of Mental Disorders (DSM-5) and was replaced with two separate diagnoses: somatic symptom disorder (SSD; diagnostic code F45.1) and illness anxiety disorder (IAD; diagnostic code F45.21). These conditions are characterized as disorders involving excessive concern with one's health in the absence of objective, verifiable evidence of a health condition. ${ }^{5}$ Although these two terms are frequently used interchangeably, the two diagnoses have subtle differences.

The core feature of SSD is the presence of one or more somatic symptoms that are distressing or result in significant disruption of daily life. The criteria for SSD are as follows:

- Excessive thoughts, feelings, or behaviors related to the somatic symptoms or associated health concerns as manifested by at least one of the following:

- Disproportionate and persistent thoughts about the seriousness of one's symptoms

- Persistently high level of anxiety about health or symptoms

- Excessive time and energy devoted to these symptoms or health concerns

- Although a somatic symptom may not be continuously present, the state of being symptomatic is persistent (typically $>6$ months). ${ }^{5}$

The core feature of IAD is a preoccupation with having or acquiring a serious, undiagnosed medical illness. The criteria for IAD are as follows:

- The individual is preoccupied with having or acquiring a serious illness.

- Somatic symptoms are not present or, if present, are only mild in intensity. If another medical condition is present or if there is a high risk for developing a medical condition (eg, strong family history is present), the preoccupation is clearly excessive or disproportionate. 
- The individual has a high level of anxiety about health and is easily alarmed about personal health status.

- The individual performs excessive health-related activities (eg, repeatedly checks his/her own body for signs of disease) or exhibits maladaptive avoidance (eg, avoids seeking healthcare).

- Illness preoccupations have been present for at least 6 months, but the specific illness that is feared may change over time.

- The individual's preoccupation is not better explained by another mental disorder. ${ }^{5,6}$

In the DSM-IV-TR, the estimated incidence of SSD/IAD in the general population is $1.5 \%$ and $2 \%-7 \%$ in the medical population. ${ }^{7}$ The SSD/IAD patient's consumption of healthcare expenditures and usage is estimated to be twice that of patients who do not have SSD/IAD. ${ }^{8}$

\section{SIGNS OF SOMATIC SYMPTOM DISORDER/ILLNESS ANXIETY DISORDER}

Identifying patients with SSD/IAD can be challenging because no pattern in their complaints clearly points to a known organic diagnosis or condition. Two of the authors (N. H. B and D. M.) have seen patients who complain about minor episodes of dysuria despite the absence of any findings after a negative workup. The patients who complain of pain everywhere and exhibit exaggerated reactions to physician palpation are likely suffering from SSD/IAD. Patients who are constantly examining their bodies for any sign of new symptoms are usually demonstrating excessive anxiety about their health.

The best predictor of SSD/IAD is a history of consulting multiple physicians and yet no diagnosis or effective treatment has been provided. When a patient has been evaluated by several competent allopathic doctors, including neurologists, immunologists, and allergists, and also by complementary healthcare providers, including chiropractors, acupuncturists, and homeopathic physicians, but none has been able to make a diagnosis, a diagnosis of SSD/IAD must be considered.

Another telltale sign of IAD/SSD is patient complaints that do not correspond with any physiologic pathway or known pathologic entity. The most common signs and symptoms are absent. For example, if a patient has the chief complaint of intermittent pain in both elbows or knees but has no physical, laboratory, or imaging findings to support joint pathology-especially if the patient has seen multiple practitioners - SSD/IAD should be considered. Medically unexplained complaints are often a symptom of SSD/IAD. ${ }^{9}$

According to Barsky et al, patients with SSD/IAD share a common feature: excessive and persistent health anxiety associated with significant distress and impairment with the absence of physical findings or imaging studies that can explain the patients' physical complaints. ${ }^{6}$

\section{MEDICAL ASSESSMENT}

Taking a careful history is important with all patients but is especially important with the patient with potential SSD/IAD.

\section{Physical Examination}

Characteristically, the absence of physical findings, particularly after serial examinations, supports a diagnosis of
SSD/IAD. However, the patient must receive a thorough physical examination to ensure he/she has no underlying organic problem. Conducting a mental status examination, taking the history of the present illness, and performing a review of systems are necessary.

Generally, patients with SSD/IAD are well-groomed and not grossly disheveled. They are cooperative, yet ill at ease and not easily reassured. Patients may demonstrate anxiety through moist hands, perspiring forehead, and wringing their hands. Patients with SSD/IAD are often restless and frequently shift their posture.

For patients with SSD/IAD, the physical examination does not reveal any findings that correlate with their complaints. With neurologic testing to touch and pinprick, anatomic or normal dermatome distribution may be absent; all the authors have seen patients who complain of a glove and stocking distribution to their pain. Patients may overreact to the physical examination or to testing; for example, they may have a disproportionate response to simple tests such as venipuncture.

The bottom line for identifying a patient with SSD/IAD is complaints that are out of proportion to the physical findings and that have no pathophysiologic explanation.

\section{Evaluation}

Patients with SSD/IAD have usually seen other physicians, so imaging and laboratory reports may be available. Reviewing previous studies is important; limit additional testing only to rule out a possibly missed organic condition. Using noncontrast imaging such as sonography instead of computed tomography scans may be indicated to minimize cost and radiation exposure.

\section{Management}

A MEDLINE search for treatment of SSD/IAD revealed a paucity of scientific studies. In their review, Khare and Srivastava state, "The treatment modules for hypochondriasis are unfortunately few and limited." 10 However, a few investigators have tried to provide guidelines and helpful suggestions.

Recognizing that patients with SSD/IAD are suffering is important. Sources of frustration that add to patients' anxiety are pain and the discomfort of not knowing what the problem is. Once a practitioner has seen a patient with SSD/IAD, one option is to keep the situation stable by continuing to see the patient and to treat him/her kindly but perhaps with tactful benign neglect. However, not all physicians are comfortable with continuing to see patients with SSD/IAD, so a second option is to try to refer them to another provider who may have experience with SSD/IAD. A third option is to try to help the patient understand the nature of the condition in the hope that it can be alleviated. This approach is challenging and time consuming in clinical practice, but if the physician is successful, the rewards can be substantial.

If the third option is selected, saying, "We have done all the testing. There is nothing wrong; it's all in your head," is unlikely to be productive or convincing to a patient with $S S D / I A D$, and because the patient has likely heard the same statement from previous healthcare providers, this approach is likely to antagonize and upset him/her. If the clinician chooses to help a patient recognize that he/she has $S S D / I A D$, the first challenge is to get the patient to agree that 
he/she does not have a physical ailment. Time and effort are required to work through the patient's anxiety, reach acceptance, and then move on to treatment of the underlying issue. Resistance is common, not only from the patient but also from the patient's family who also believe that clinicians have simply failed to make a proper diagnosis of an organic ailment. Despite the physician's best efforts, failure is common in this first step. However, several approaches can be used to bridge this gap with the patient.

Initially, reassurance that all proper steps have been taken to rule out somatic disease is very important. Many patients need to be reassured that they have no evidence of cancer. The physician must acknowledge the patient's pain and explain that the normal state of the human body is not to be pain-free, so pain is not always an indication of disease and is not necessarily attributable to a serious condition. Explaining that everyone suffers from aches and pains as a normal function of living and aging is often helpful.

After reassurance, medications and behavior modification methodologies have been used with varying degrees of success. Noting that "there are no previous treatment studies for these disorders" (referring to SSD/IAD), Hedman and coworkers described success with three cognitive behavioral therapy approaches: therapist-guided, internet-guided, and unguided bibliotherapy. ${ }^{11}$

Data about medical therapy are limited as well. However, several small studies and case studies suggest that the use of antidepressants, including selective serotonin reuptake inhibitors, imipramine, amitriptyline, and monoamine oxidase inhibitors, may be beneficial for the treatment of SSD/IAD. ${ }^{12,13}$ A randomized controlled trial published by Fallon et al in 2017 evaluated the treatment of SSD/IAD with fluoxetine, cognitive behavioral therapy, and a combination of the two treatments vs placebo. ${ }^{14}$ The findings support fluoxetine as a safe and efficacious treatment for hypochondriasis. However, continued research is needed before guidelines for the treatment of SSD/IAD can be recommended.

Where does that leave the physician-patient relationship? Begin by establishing a firm therapeutic alliance with the patient. Be empathetic about the patient's condition even if the root cause cannot be identified. Educate the patient regarding the manifestations of SSD/IAD. This is a difficult action step as the physician is likely to meet resistance when confronting the patient with a diagnosis of SSD/IAD. The compassionate doctor offers consistent reassurance that he/she understands the patient's complaints, but the workup has been thorough, and the patient does not have a serious condition. Try to optimize the patient's ability to cope with the symptoms rather than trying to eliminate the symptoms. Physicians need to avoid the temptation to perform highrisk, low-yield, invasive procedures. Touching a patient with a scalpel when the likelihood of solving the complaint is very low will likely result in a disappointed patient. Finally, when caring for a patient with SSD/IAD, close collaboration among all providers is mandatory to prevent investigative duplication. The management of the patient with SSD/IAD is time consuming, and the psychological rewards for the physician are minimal as the patient continues to have pain and discomfort. However, the art of medicine comes into play in this scenario; having frequent follow-up visits may help reassure the patient that the clinician cares about his/her concerns and may help prevent the patient from doctor shopping.

\section{CONCLUSION}

Numerous patients who see physicians in a variety of medical specialties have no serious organic disease but remain convinced they are harboring a yet-to-be-diagnosed condition. These individuals with hypochondriasis or SSD/IAD are suffering and are a time-consuming challenge to the clinician. Physicians must first rule out significant organic disease and then provide treatment that consists of reassurance, counseling, medication, and when possible, referral to a mental health specialist. The paucity of large studies or trials is evident upon reviewing the literature. SSD/IAD is an area of medical practice that could greatly benefit from serious research and clinical trials.

\section{ACKNOWLEDGMENTS}

Dr Nemeroff has consulted with the following companies: Xhale, Takeda, Taisho Pharmaceutical Inc., Prismic Pharmaceuticals, Bracket (Clintara), Total Pain Solutions, Gerson Lehrman Group Healthcare \& Biomedical Council, Fortress Biotech, Sunovion Pharmaceuticals Inc., Sumitomo Dainippon Pharma, Janssen Research \& Development LLC, Magstim, Inc., Navitor Pharmaceuticals, Inc., TC MSO, Inc., and Intra-Cellular Therapies, Inc. Dr Nemeroff is a stockholder in the following companies: Xhale, Celgene, Seattle Genetics, Abbvie, OPKO Health, Inc., Network Life Sciences Inc., Antares, and BI Gen Holdings, Inc. Otherwise, the authors have no financial or proprietary interest in the subject matter of this article.

\section{REFERENCES}

1. Hypochondriasis. Drugs.com. www.drugs.com/health-guide/ hypochondriasis.html. Updated September 10, 2018. Accessed April 10, 2019.

2. Hypochondria. Merriam-Webster Dictionary. www.merriamwebster.com/dictionary/hypochondria. Accessed April 10, 2019.

3. Illness anxiety disorder. Mayo Clinic. www.mayoclinic.org/ diseases-conditions/illness-anxiety-disorder/symptoms-causes/ syc-20373782. Published June 6, 2018. Accessed April 10, 2019.

4. Hypochondria. Cambridge Dictionary. dictionary.cambridge. org/dictionary/english/hypochondria. Accessed April 10, 2019.

5. American Psychiatric Association. Diagnostic and Statistical Manual of Mental Disorders. 5th ed. Washington, DC: American Psychiatric Publishing; 2013.

6. Barsky AJ, Peekna HM, Borus JF. Somatic symptom reporting in women and men. J Gen Intern Med. 2001 Apr;16(4):266-275.

7. Tsaman A, Kay J, Liebman JA, eds. Psychiatry, Vol 2. 1st ed. Philadelphia, PA: WB Saunders; 1997.

8. Taylor L, Brooks B. Strategies to manage the patient with health anxiety. JAAPA. 2013 Jan;26(1):61-62.

9. Kurlansik SL, Maffei MS. Somatic symptom disorder. Am Fam Physician. 2016 Jan 1;93(1):49-54.

10. Khare S, Srivastava MN. Validity of current treatment protocols to overcome hypochondriasis. J Clin Diagn Res. 2017 Jan;11(1):VE01-VE04. doi: 10.7860/JCDR/2017/22509.9262.

11. Hedman E, Axelsson E, Andersson E, Lekander M, Ljótsson B. Exposure-based cognitive behavioural therapy via the internet and as bibliotherapy for somatic symptom disorder and illness anxiety disorder: randomized controlled trial. $\mathrm{Br} J$ Psychiatry. 2016 Nov;209(5):407-413. doi: 10.1192/bjp.bp.116.181396.

12. Somashekar B, Jainer A, Wuntakal B. Psychopharmacotherapy of somatic symptoms disorders. Int Rev Psychiatry. 2013 Feb;25(1):107-115. doi: 10.3109/09540261.2012.729758. 
13. Kleinstäuber $M$, Witthöft $M$, Steffanowski A, van Marwijk H, Hiller W, Lambert MJ. Pharmacological interventions for somatoform disorders in adults. Cochrane Database Syst Rev. 2014 Nov 7;(11):CD010628.

doi: 10.1002/14651858.CD010628.pub2.
14. Fallon BA, Ahern DK, Pavlicova M, Slavov I, Skritskya N, Barsky AJ. A randomized control trial of medication and cognitive-behavioral therapy for hypochondriasis. Am J Psychiatry. 2017 Aug 1;174(8):756-764. doi: 10.1176/appi.ajp.2017.16020189. 